\title{
Spin polarization contrast observed in GaAs by force-detected nuclear magnetic resonance
}

\author{
Kent R. Thurber \\ U.S. Army Research Laboratory, AMSRL-SE-EM, Adelphi, Maryland 20783 and Center for Superconductivity Research, \\ Department of Physics, University of Maryland, College Park, Maryland 20742 \\ Lee E. Harrell \\ Department of Physics, U.S. Military Academy, West Point, New York 10996 \\ Raúl Fainchtein \\ Johns Hopkins University Applied Physics Laboratory, 11100 Johns Hopkins Road, Laurel, Maryland 20723 \\ Doran D. Smith \\ U.S. Army Research Laboratory, AMSRL-SE-EM, Adelphi, Maryland 20783
}

(October 30, 2018)

\begin{abstract}
We applied the recently developed technique of force-detected nuclear magnetic resonance (NMR) to observe ${ }^{71} \mathrm{Ga},{ }^{69} \mathrm{Ga}$, and ${ }^{75} \mathrm{As}$ in GaAs. The nuclear spin-lattice relaxation time is $21 \pm 5 \mathrm{~min}$ for ${ }^{69} \mathrm{Ga}$ at $\sim 5 \mathrm{~K}$ and 4.6 Tesla. We have exploited this long relaxation time to first create and then observe spatially varying nuclear spin polarization within the sample, demonstrating a new form of contrast for magnetic resonance force microscopy (MRFM). Such nuclear spin contrast could be used to indirectly image electron spin polarization in GaAs-based spintronic devices.
\end{abstract}

76.60.Pc,81.05.Ea

Due to its long spin coherence lifetime and scattering length 1 , 2 GaAs has received attention as a potential material for spintronic devices that exploit the spin degree of freedom of the electron. 3 For example, B. Jonker et al. 1 have demonstrated a scheme for the injection of spin-polarized carriers into GaAs/AlGaAs heterostructures via an epitaxial layer of magnetic ZnMnSe. A technique that can image the electron spin polarization at any arbitrary point in the device would be very useful for determining whether spin flips are occurring at an interface or in the bulk material. Short device transit times make direct imaging of the electron polarization difficult. However, dynamic nuclear polarization 16 provides an indirect route to the time-averaged electron polarization. In dynamic nuclear polarization, carriers become trapped on defects and transfer their polarization to nearby nuclei via the hyperfine interaction. The induced nonequilibrium polarization of the nuclear lattice persists for minutes, so it could be measured by a magnetic resonance imaging technique with adequate sensitivity and resolution, such as MRFM, a scanned probe microscopy based on force-detected magnetic resonance.

In this letter, we demonstrate the first steps towards indirect electron polarization imaging using force-detected NMR. We create and then observe nuclear spin polarization contrast in GaAs. Figure 1 shows force-detected NMR observations of ${ }^{71} \mathrm{Ga},{ }^{69} \mathrm{Ga}$, and ${ }^{75} \mathrm{As}$, increasing the number of observed isotopes from four $\left({ }^{1} \mathrm{H},{ }^{19} \mathrm{~F},{ }^{59} \mathrm{Co}\right.$, $\left.{ }^{23} \mathrm{Na}\right) 811$ to seven. Further, this demonstrates the observation of multiple isotopes within a single sample using force-detected NMR techniques. In addition, we used off resonance cantilever excitation, which allows the mea- surement of nuclear isotopes with small gyromagnetic ratio, $\gamma$, without requiring large radio frequency (RF) magnetic fields.

In force-detected NMR, the sample is mounted on a micro-cantilever in an applied magnetic field with a magnetic field gradient arising from a nearby magnetic particle. The field gradient exerts a force on the magnetized sample. The magnetization of a particular nuclear is $\rho_{-}$ tope is isolated by using adiabatic rapid passage (ARP) 12 to cyclically invert its magnetic moment at a frequency $f_{A R P}$. The signal appears as a driven cantilever oscillation. The magnetic field gradient restricts the NMR resonance to a sensitive slice of the sample, so magnetic resonance imaging is possible.13

The sample and cantilever form a mechanical resonator with resonance frequency $f_{c}$ and quality factor $Q$. The amplitude $A$ of the cantilever response depends on $f_{A R P}$ and the amplitude $F$ of the driving force. For $f_{A R P} \ll f_{c}$, the cantilever response is essentially the zero frequency response, $A=F / k$, where $k$ is the spring constant of the cantilever. When driven on resonance, the cantilever response is enhanced by a factor of $Q$. Well-understood restrictions limit the frequency at which spins can be flipped by ARP. In particular,

$$
\Omega \leq \frac{\left(\gamma B_{1}\right)^{2}}{4 f_{A R P}}
$$

where $\gamma$ is the gyromagnetic ratio, and $\Omega$ is the amplitude of the frequency modulation of the radio frequency (RF) magnetic field $B_{1}$. On resonance excitation of the cantilever $\left(f_{A R P}=f_{c}\right)$ can require a large $\mathrm{RF}$ field $B_{1}$ 
if $\gamma$ is small. Our experiment demonstrates the utility of off resonance cantilever excitation $\left(f_{A R P}<f_{c}\right)$. By eliminating the need to satisfy the adiabatic rapid passage condition at the cantilever resonance frequency, we reduced our required RF magnetic field by almost a factor of 8 (and thus reduced our RF power needs by a factor of 60 !)

Our sample is a $3 \mu \mathrm{m}$ epilayer of GaAs, doped at $0.6 \times 10^{18} \mathrm{~cm}^{-3} \mathrm{Si}$ and $2.0 \times 10^{18} \mathrm{~cm}^{-3} \mathrm{Be}$, grown on a AlAs lift-off layer and released using HF. A hand-cleaved irregular rectangle of this material $(\approx 210 \mu \mathrm{m} \times 150 \mu \mathrm{m})$ is attached to the cantilever $(\mathrm{k} \sim 0.05 \mathrm{~N} / \mathrm{m}) \mathrm{t}$ using silverfilled epoxy. The cantilever is coated with $0.2 \mu \mathrm{m}$ of $\mathrm{Au}$ on each side to enhance thermal conductivity. The loaded cantilever has $f_{c}=1.034 \mathrm{kHz}$ and $Q=154$ at $5 \mathrm{~K}$ in the presence of He exchange gas. The cryostat and detection electronics are described in Ref. 15. The magnetic gradient source is a $250 \mu \mathrm{m}$ diameter Fe cylinder positioned $\sim 35 \mu \mathrm{m}$ from the sample. Because the sample is thin, the sensitive slices are circular arcs that increase in radius with applied magnetic field at fixed RF frequency as shown in Fig. 2(a).

A tuned and matched $11 / 2$ turn, $710 \mu \mathrm{m}$ diameter coil generates the 4 gauss transverse RF magnetic field. The $\mathrm{RF}$ waveform is generated by a Hewlett-Packard model HP $8642 \mathrm{~B}$ source driven by a $f_{A R P}=16.6 \mathrm{~Hz}$ triangle wave frequency modulation, with subsequent amplification. The triangle wave is offset such that only positive frequency deviations up to $88 \mathrm{kHz}$ (i.e. $\Omega=44 \mathrm{kHz}$ ) are obtained. The motion of the cantilever is detected by a temperature-tuned fiber optic interferometen 16 with the fiber rigidly epoxied to the cantilever support. The signal is demodulated with a lock-in amplifier referenced to the frequency modulation signal.

Figure 1 displays a force-detected NMR spectrum of our sample observed by sweeping the applied magnetic field at a fixed RF center frequency of $51.56 \mathrm{MHz}$. All three isotopes are clearly resolved with cantilever displacements of $\sim 1.3$ picometers. The large width of the NMR peaks represents the spatial extent of the sample. As illustrated in Fig. 2(a), each point in the NMR line of each isotope represents the signal from a ring of the sample. On the axis of the cylindrical iron magnet, the magnetic field is strongest, which means the applied external magnetic field required for magnetic resonance is less. Thus, the points at lower applied magnetic field represent the sample near the center of the magnet.

Using the observed maximum offset of the signal (0.8 Tesla) from the nominal resonance fields-and the saturation magnetization for iron (2.18 Tesla) 17 we can calculate the magnetic field gradient and the geometry of the sensitive slice. As an example, for the region shown in Fig. 2(a), the volume of a typical sensitive slice is 870 $\mu \mathrm{m}^{3}$ with a magnetic field gradient of $8600 \mathrm{~T} / \mathrm{m}$. Using thermal polarization of ${ }^{69} \mathrm{Ga}$ at $5 \mathrm{~K}$ gives an estimated $3.4 \mathrm{pm}$ cantilever excitation over the 10 second lock-in integration period. Analysis of ${ }^{75} \mathrm{As}$ and ${ }^{71} \mathrm{Ga}$ gives similar results. Our experimental signal is $\sim 2.5$ times smaller than this estimate. Recent tests have suggested that RF noise may still be a problem.

In our $0.1 \mathrm{~Hz}$ bandwidth, we observe $0.2 \mathrm{pm}$ rms cantilever noise. Assuming a frequency independent force spectral power density, the root mean square thermal excitation of the cantilever in a given narrow bandwidth $\Delta f$ well below resonance,

$$
x_{\mathrm{rms}, \text { thermal }}=\left(\frac{2 k_{B} T \Delta f}{\pi k f_{c} Q}\right)^{1 / 2}
$$

is smaller than the on resonance thermal noise by a factor of $Q$. Interestingly, the observed signal is also a factor of $Q$ smaller in the low frequency regime relative to an on resonance excitation, so the thermal signal-to-noise ratio is independent of $f_{A R P} .18$ Using equation (2) we estimate $x_{\mathrm{rms} \text {, thermal }}=0.02 \mathrm{pm}$. Currently, our noise is dominated by the interferometer electronics.

By averaging lock-in traces, we were able to see the time dependent decay, $\exp \left(-t / \tau_{m}\right), 19$ of the nuclear magnetization when cyclically flipped by our ARP sequence. The observed $\tau_{m}$ values are $7 \mathrm{~s}$ for ${ }^{75} \mathrm{As}$ (applied fields 6.28 to 6.44 Tesla), $20 \mathrm{~s}$ for ${ }^{69} \mathrm{Ga}$ (4.28 to 4.44 Tesla), and $30 \mathrm{~s}$ for ${ }^{71} \mathrm{Ga}$ (3.26 to 3.42 Tesla) for the data displayed in Fig. 1. In our experiment, the driven relaxation time of the nuclear magnetization depends on the adiabatic condition for the spin flips (Eq. 1). Larger $\gamma$ and $B_{1}$ and smaller $f_{A R P}$ increase the relaxation time, $\tau_{m}$.

For future work, we are interested in the nuclear spinlattice relaxation time, $T_{1}$, of our sample. To determine $T_{1}$ for ${ }^{69} \mathrm{Ga}$, we destroyed the magnetization by applying our ARP sequence for $5 \tau_{m}$, and then allowed it to recover at an applied field of $4.0 \mathrm{~T}$ (total field 4.5 to $4.7 \mathrm{~T}$ ) for a range of delay times before observing the NMR signal. Fitting the data to a single exponential gives $T_{1}=21+5$ min, in agreement with previously published results.

The relatively long $T_{1}$ of GaAs at low temperatures allows us to create and then measure spin polarization contrast in the sample before the spins relax back to thermal equilibrium. Figure 2(b) shows an example of spin polarization contrast created by using a single ARP sweep to invert the nuclear polarization in all but a narrow slice of the sample. In the applied field range 4.39 to 4.45 Tesla, the ${ }^{69} \mathrm{Ga}$ spin polarization is still aligned with the magnetic field. Outside of this field range, the force signal is negative relative to the thermal signal, indicating that the magnetization has been flipped to point opposite to the applied magnetic field. Figure 2(a) illustrates the spatial dependence of the corresponding spin polarization contrast in the sample. This data demonstrates conclusively that cycling spins by ARP in one volume of the sample does not disturb the polarization in nearby out of resonance sample volumes, an important consideration for efficient imaging of nonequilibrium nuclear polarization.

In conclusion, we applied force-detected NMR techniques to GaAs, demonstrating force-detection of spin polarization contrast. A spin-lattice relaxation time of 
$21 \pm 5$ min was observed for ${ }^{69} \mathrm{Ga}$. This work significantly increases the total number of isotopes observed and reported in the force-detected NMR literature. We used off cantilever resonance force-detected NMR, which allows the measurement of nuclei with small $\gamma$ at reasonable RF fields. In fact, ${ }^{75} \mathrm{As}$ has the smallest $\gamma$ measured by force-detected NMR to date.

This work was supported in part by the U.S. Army Research Laboratory Microelectronics Research Collaboration Program, Grant No. DAAL01-95-2-3530, the DARPA Defense Science Office, and the U.S. Military Academy/Army Research Laboratory Faculty Research Program. The sample was grown and processed by Peter Newman and Monica Taysing-Lara. We would like to thank John A. Marohn, James G. Kempf, and Daniel Weitekamp for many helpful discussions and suggestions.

${ }^{1}$ J. M. Kikkawa and D. D. Awschalom, Phys. Rev. Lett. 80, 4313 (1998).

${ }^{2}$ D. Hägele, M. Oestreich, W. W. Rühle, N. Nestle, K. Eberl, Appl. Phys. Lett. 73, 1580 (1998).

${ }^{3}$ Philip Ball, Nature 404, 918 (2000).

${ }^{4}$ B. T. Jonker, Y. D. Park, B. R. Bennett, H. D. Cheong, G. Kioseoglou, and A. Petrou, Phys. Rev. B 62, 8180 (2000).

${ }^{5}$ V. L. Berkovits, A. I. Ekimov, and V. I. Safarov, Sov. Phys. JETP 38, 169 (1974).

${ }^{6}$ M. Dobers, K. v. Klitzing, J. Schneider, G. Weimann, and K. Ploog, Phys. Rev. Lett. 61, 1650 (1988).

${ }^{7}$ J. A. Sidles, J. L. Garbini, K. J. Bruland, D. Rugar, O. Züger, S. Hoen, and C. S. Yannoni, Rev. Mod. Phys. 67, 249 (1995).

${ }^{8}$ D. Rugar, O. Züger, S. Hoen, C. S. Yannoni, H. M. Vieth, R. D. Kendrick, Science 264, 1560 (1994).

${ }^{9}$ K. Wago, O. Züger, R. Kendrick, C. S. Yannoni, and D. Rugar, J. Vac. Sci. Technol. B 14, 1197 (1996).

${ }^{10}$ D. V. Pelekhov, P. C. Hammel, M. M. Midzor, and M. L. Roukes, Bull. Am. Phys. Soc. 44, 542 (1999).

${ }^{11}$ Rieko Verhagen, Cees Hilbers, Arno Kentgens, and Herman Van Kempen, Bull. Am. Phys. Soc. 46, 284 (2001).

12 C. P. Slichter, Principles of Magnetic Resonance, 3rd edition, (Springer-Verlag, Berlin, 1990), pp. 22-25.

${ }^{13}$ O. Züger, S. T. Hoen, C. S. Yannoni, and D. Rugar, J. Appl. Phys. 79, 1881 (1996).

${ }^{14}$ MLCT-NOHW Microcantilevers, Park Scientific Instruments, Sunnyvale, CA.

${ }^{15}$ Doran D. Smith, John A. Marohn, and Lee E. Harrell, Rev. Sci. Instrum. 72, 2080 (2001).

${ }^{16}$ K. J. Bruland, J. L. Garbini, W. M. Dougherty, S. H. Chao, and S. E. Jensen, Rev. Sci. Instrum. 70, 3542 (1999).

${ }^{17}$ R. M. Bozorth, Ferromagnetism, (Van Nostrand, New York, 1951), pg. 54.

${ }^{18}$ Kelly Bruland, "Dynamics and Control of Magnetic Resonance Force Microscopy," Ph.D. Dissertation, University of Washington, 46 (1997).
${ }^{19}$ K. Wago, D. Botkin, C. S. Yannoni, and D. Rugar, Phys. Rev. B 57, 1108 (1998); Garett M. Leskowitz, Louis A. Madsen, Daniel P. Weitekamp, Solid State Nuclear Magnetic Resonance 11, 73 (1998).

FIG. 1. Force-detected NMR signal of GaAs as a function of applied magnetic field at constant RF center frequency $51.56 \mathrm{MHz}$. Spins were flipped at $f_{A R P}=16.6 \mathrm{~Hz}$ with ARP frequency modulation of $\pm 44 \mathrm{kHz}$. Lock-in detection was used with a $1 \mathrm{~s}$ time constant, and $10 \mathrm{~s}$ of data were integrated. ${ }^{71} \mathrm{Ga},{ }^{69} \mathrm{Ga}$, and ${ }^{75} \mathrm{As}$ are well resolved. Peak widths are largely determined by the spatial extent of the sample and the magnetic field gradient. Offset of the signal from the nominal Larmor frequency is due to the extra magnetic field of the gradient source. A linear background has been subtracted from the data for clarity.

FIG. 2. (a) Illustration of the induced nuclear spin polarization contrast (sample, cantilever and iron cylinder (magnet) drawn to scale - sample and cantilever thickness exaggerated). The interferometer fiber and RF coil are omitted for clarity. The circular arc on the sample indicates the 11 $\mu \mathrm{m}$ wide region where the ${ }^{69} \mathrm{Ga}$ spin polarization is aligned with the magnetic field, while the ${ }^{69} \mathrm{Ga}$ spins in the rest of the sample are aligned opposite the applied magnetic field. (b) Force-detected ${ }^{69} \mathrm{Ga}$ NMR signal as a function of applied magnetic field. The thermal polarized data $(\square)$ is shown expanded from Fig. 1. The second set of data $(\bullet)$ was measured after the ${ }^{69} \mathrm{Ga}$ spin polarization was patterned by an ARP sweep. A single ARP sweep inverted the nuclear polarization for most of the sample except for the applied field region 4.39 to 4.45 Tesla when the RF field was turned off (illustrated in part a). In order to enhance the contrast, the nuclear spins were thermalized at 8 Tesla prior to patterning, the lock-in was used with a $0.3 \mathrm{~s}$ time constant, and $5 \mathrm{~s}$ of data was integrated. 


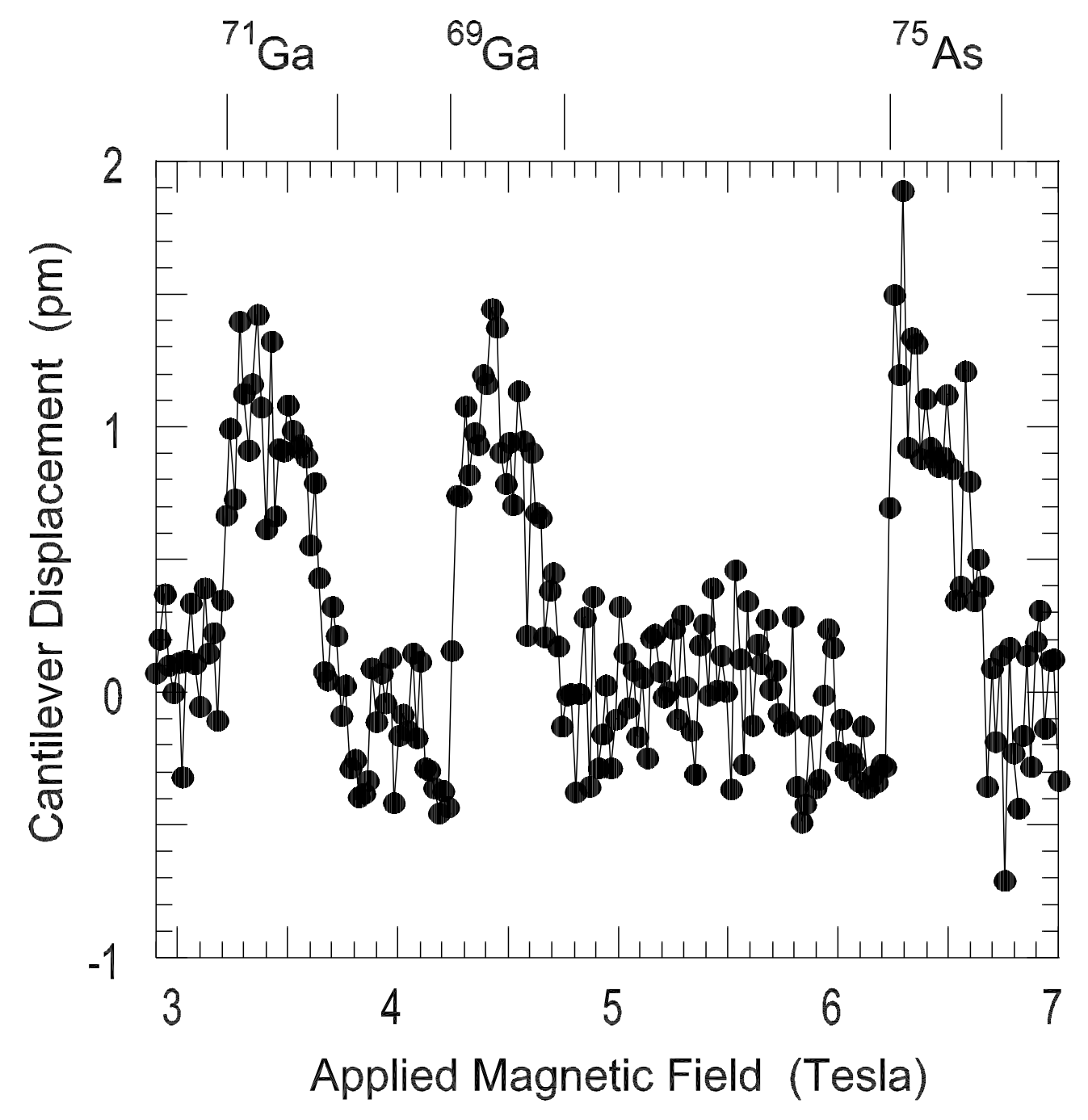



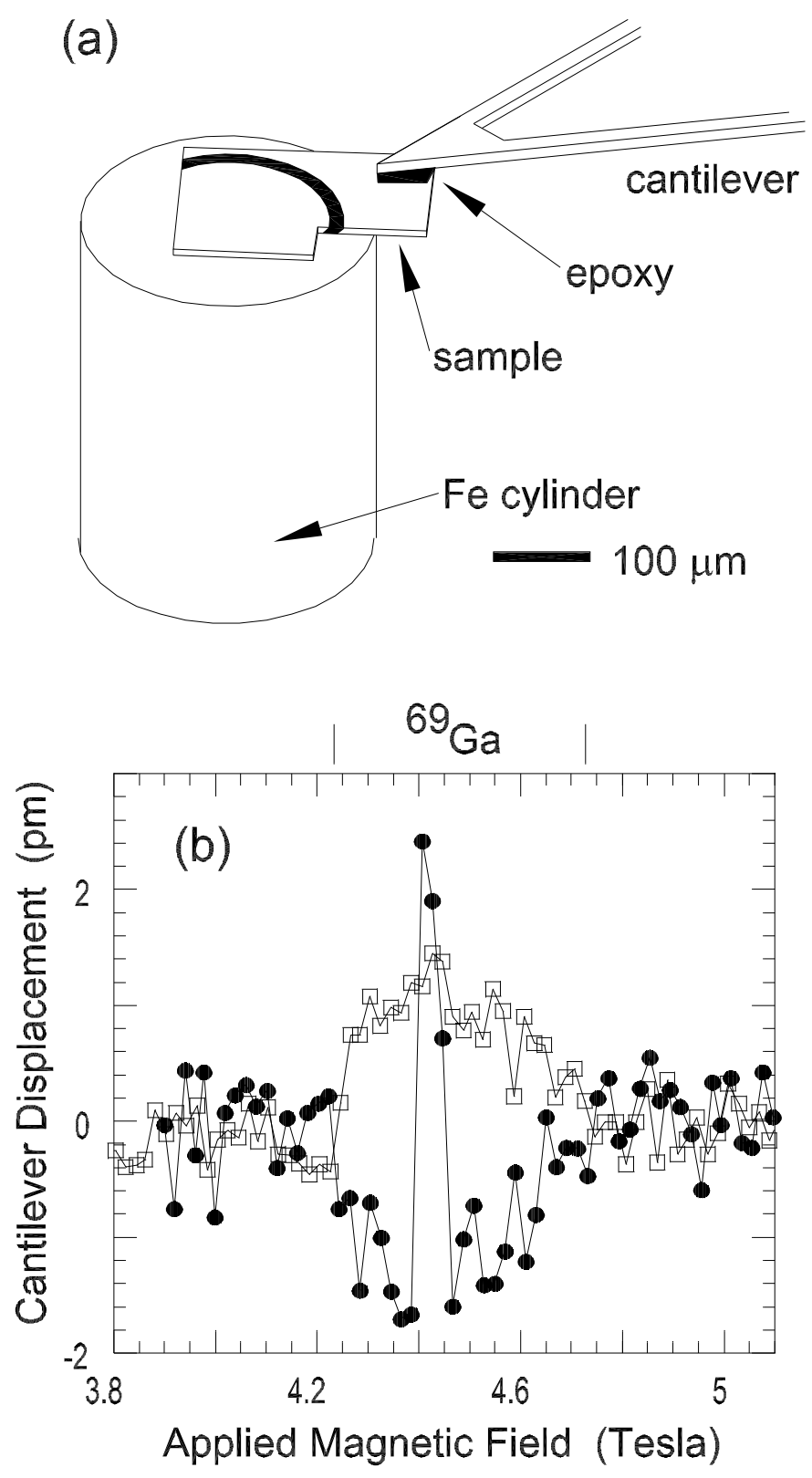